\title{
IMATUROS DE MOSQUITOS (DIPTERA, CULICIDAE) DE ÁREAS URBANA E RURAL NO NORTE DO ESTADO DO PARANÁ, BRASIL ${ }^{1}$
}

\begin{abstract}
Allan Martins da Silva ${ }^{2}$
ABSTRACT

IMMATURES OF MOSQUITOES (DIPTERA, CULICIDAE) IN URBAN AND RURAL ENVIRONMENTS OF NORTHERN PARANÁ STATE, BRAZIL. The results of larval collections of mosquitoes from artificial containers and natural breeding at urban and rural areas carried out at Sertaneja, northern State Paraná, Brazil, from February to April, 1995, are presented. Among the 4534 immature forms collected, belonging to 21 species or species-groups, the species with higher density were Aedes aegypti (Linnaeus, 1762), Aedes albopictus (Skuse, 1894), Culex quinquefasciatus Say, 1823 and Limatus durhami Theobald, 1901.
\end{abstract}

KEYWORDS. Culicidae, larvae, vectors, Brazil.

\section{INTRODUÇÃO}

Os ecossistemas antrópicos são diferentes dos naturais por apresentarem feições próprias (Clapham Jr. apud FoRATTINI, 1992). Tanto os ambientes urbanos quanto o rural, sofrem modificações profundas devido à atividade humana, a qual influencia formas imaturas de culicídeos ditos sinantrópicos que tendem a ocupar determinados tipos de criadouros, na sua maioria, artificiais.

No Estado do Paraná (Brasil), estudos foram realizados na intenção de identificar o comportamento de imaturos de mosquitos frente ao ambiente modificado pelo homem: Andrade \& Rachou (1954), Lopes et al. (1987), Silva \& Menezes (1996) em área rural; SiLva \& Lopes (1985), Luz et al. (1987), Lopes et al. (1993) em área urbana e Lozover \& LuZ (1976a,b), nos dois ambientes.

Apresenta-se os resultados das coletas de larvas realizadas no município de Sertaneja, norte do Paraná, expondo de forma quali- e quantitativa a composição da fauna de Culicidae de criadouros artificiais e naturais em áreas urbana e rural.

1. Financiado pela Fundação Nacional de Saúde.

2. Secretaria de Estado da Saúde do Paraná, 19ª Regional de Saúde, Seção de Ação Sobre o Meio, Caixa Postal 214, 86400-000, Jacarezinho, PR, Brasil. 


\section{MATERIAL E MÉTODOS}

O município de Sertaneja está localizado no terceiro planalto do Estado do Paraná, na zona fisiográfica do Norte $\left(23^{\circ} 01^{\prime} \mathrm{S}, 50^{\circ} 47^{\prime} \mathrm{W}\right)$. A Mata Tropical nesta região foi inicialmente substituída, em sua maior parte, pela cultura cafeeira, hoje ocupada por rotação de culturas de milho, trigo e soja (Wons, 1985). Foram investigadas duas áreas urbanas, a cidade de Sertaneja e o povoado de Paranagi, entre 1 de fevereiro e 24 de abril de 1995. Na área rural, foram amostrados seis pontos, com $400 \mathrm{~m}$ de raio, separados por distâncias regulares de $3 \mathrm{~km}$, nas rodovias PR 160 e PR 323, entre os quilômetros 021 e $0-8$, respectivamente.

As pesquisas realizadas nas áreas urbanas cobriram residências, terrenos baldios, cemitério e outros possíveis pontos para o desenvolvimento larvário de mosquitos, como postos de combustível, borracharias e depósitos de material de construção. $\mathrm{Na}$ área rural, incluíram-se residências, campos de cultivo, escola rural, aviário e posto de fiscalização. Todos os reservatórios contendo água foram observados, artificiais ou naturais. Os imaturos foram recolhidos com peneira de malha fina, aplicada em vários lances, objetivando coletar maior número de indivíduos e acondicionados em pequenos tubos de vidro contendo etanol a 70\%, devidamente etiquetados, transportados para o laboratório, analisados e depositados na coleção da 19ํㅗㄹ Regional de Saúde de Jacarezinho, Paraná.

A identificação dos exemplares foi realizada com bibliografia de LANE (1953a,b); ForatTini (1962, 1965a,b); Consoli \& Lourenço-De-Oliveira (1994). A abreviação dos gêneros segue Reinert (1975).

\section{RESULTADOS E DISCUSSÃO}

Foram coletadas nos dois ambientes, rural e urbano, 4534 formas imaturas de culicídeos de 21 espécies ou grupos de espécies distribuídas em oito gêneros, arroladas segundo GUIMARÃES (1997) (tab. I). As espécies com maior representatividade, coletadas nasduasárea, Ae. aegypti $43 \% \mathrm{e}$ Ae. albopictus 33,8\% foram encontradas apenas em recipientes artificiais, assim como Cx. quinquefasciatus e Li. durhami, representando respectivamente $16,1 \%$ e $2,3 \%$ (tabs. II, III). O ambiente urbano contribuiu com $86,5 \%$ dos imaturos coletados e dentre eles, os mais representativos foram Ae. aegypti (49,4\%), Ae. albopictus $(31,4 \%)$ e Cx. quinquefasciatus (16,0\%). Li. durhami, An. argyritarsis, Cx. aff. declarator, Culex sp. do grupo coronator, Ae. fluviatilis e An. triannulatus totalizaram 123 imaturos, correspondendo a 3,2\% do total coletado.

Para os 610 imaturos coletados no ambiente rural (tab. III), além da maioria das espécies citadas para o ambiente urbano, foram encontradas Ur. nataliae, Cx. eduardoi, Ur. lowii, Cx. chidesteri, Cx. bigoti, Cx. (Melanoconion) spp., An. evansae, Ae. scapularis, Ps. confinnis, Toxorhynchites sp. e Sabethes sp., sendo esta última coletada apenas uma vez, em lata pequena, abandonada na margem de uma mata residual. $\mathrm{O}$ gênero Aedes contribuiu com 312 espécimens (51,1\%), Culex 163 (26,7\%), Limatus 69 (11,3\%), Uranotaenia 44 (7,2\%), Anopheles 17 (2,8\%), Psorophora 2, Sabethes 2, Toxorhynchites 1. Neste ambiente verificou-se a predominância do Ae. albopictus sobre outras espécies em criadouros artificiais, seguida por Cx. quinquefasciatus e Li. durhami.

Aedes aegypti foi encontrada colonizando ampla variedade de recipientes em área urbana, sem mostrar preferência por qualquer um deles. Vaso de plantas, pneu e recipiente de plástico ou metal reuniram $69,6 \%$ dos exemplares coletados desta espécie, coincidindo com o observado por Lopes et al. (1993). Entretanto, na área rural a sua presença esteve restrita a poucos recipientes.

Aedes albopictus é uma espécie exótica, e assim como Ae. aegypti, poderá vir a ter importância epidemiológica. Isto se deve à sua valência ecológica que a possibilita colonizar com sucesso o ambiente urbano e o rural, utilizando criadouros naturais e 
Tabela I. Espécies, número e percentagem de imaturos de Culicidae coletados nas áreas urbana e rural de Sertaneja, PR, entre $1^{\circ}$ de fevereiro e 24 de abril de 1995.

\begin{tabular}{lrr}
\hline \multicolumn{1}{c}{ Espécies } & $\mathrm{N}$ & $\%$ \\
\hline Aedes (Stegomyia) aegypti (Linnaeus, 1762) & 1950 & 43,0 \\
Ae. (Stg.) albopictus (Skuse, 1894) & 1534 & 33,8 \\
Ae. (Ochlerotatus) fluviatilis (Lutz, 1904) & 13 & 0,3 \\
Ae. (Och.) scapularis (Rondoni, 1848) & 2 & 0,0 \\
Anopheles (Nyssorhynchus) argyritarsis Robineau-Desvoidy, 1827 & 1,0 \\
An. (Nys.) evansae (Brèthes, 1926) & 46 & 0,1 \\
An. (Nys.) triannulatus (Neiva \& Pinto, 1922) & 6 & 0,0 \\
Culex (Cux.) aff. declarator & 1 & 0,7 \\
Cx. (Cux.) chidesteri Dyar, 1921 & 31 & 0,0 \\
Cx. (Cux.) eduardoi (Casal \& Garcia, 1968) & 1 & 0,4 \\
Cx. (Cux.) quinquefasciatus Say, 1823 & 17 & 16,1 \\
Cx. (Cux.) sp. grupo coronator & 732 & 0,6 \\
Cx. (Lutzia) bigoti Bellardi, 1862 & 27 & 0,1 \\
Cx. (Melanoconion) sp. 1 & 4 & 0,1 \\
Cx. (Melanoconion) sp. 2 & 3 & 0,2 \\
Limatus durhami Theobald, 1901 & 11 & 2,3 \\
Psorophora (Grabhamia) confinnis (Lynch Arribálzaga, 1891) & 107 & 0,0 \\
Sabethes sp. & 2 & 0,0 \\
Toxorhynchites sp. & 2 & 0,0 \\
Uranotaenia (Ura.) lowii Theobald, 1901 & 1 & 0,3 \\
Ur.(Ura.) nataliae Lynch Arribálzaga, 1891 & 13 & 0,7 \\
\hline Total & 31 & 100,0 \\
\hline
\end{tabular}

Tabela II. Total de formas imaturas por espécie em diferentes tipos de criadouros artificiais, nas áreas urbanas de Sertaneja, PR, entre 1 de fevereiro e 24 de abril de 1995. Espécies: Aea, Ae. aegypti; Ael, Ae. albopictus; Aeu, Ae. fluviatilis; Ana, An. argyritarsis; Ant, An. triannulatus; Cxc, Cx. sp. grupo coronator; Cxd, Cx. aff. declarator; Cxq, Cx. quinquefasciatus; Lid, Li. durhami.

\begin{tabular}{|c|c|c|c|c|c|c|c|c|c|c|}
\hline \multicolumn{11}{|c|}{ Espécies } \\
\hline Criadouros & Aea & $\mathrm{Ael}$ & Aeu & Ana & Ant & $C x c$ & $C x d$ & $C x q$ & Lid & Total \\
\hline Pneu & 441 & 192 & & & & 2 & & 140 & 3 & 778 \\
\hline Tambor/Tanque & 97 & 58 & & 6 & 1 & 2 & 2 & 56 & & 222 \\
\hline Vaso de plantas & 570 & 552 & 13 & 5 & & 6 & 6 & 257 & & 1409 \\
\hline Material de construção & 36 & 25 & & & & & & 8 & & 69 \\
\hline Caixa d'água & 216 & 97 & & 14 & & 3 & & 45 & & 375 \\
\hline Peça de carro & 48 & 9 & & 9 & & & & 5 & 1 & 72 \\
\hline Garrafa & 21 & 32 & & & & 4 & & & 2 & 59 \\
\hline Plástico/Lata & 340 & 177 & & 1 & & & 11 & 77 & 28 & 634 \\
\hline Ralo/C. séptica/Vala/Poço & 171 & 92 & & & & & & 39 & 4 & 306 \\
\hline Total & 1940 & 1234 & 13 & 35 & 1 & 17 & 19 & 627 & 38 & 3924 \\
\hline
\end{tabular}

artificiais. Na área urbana de Sertaneja, mostrou acentuada preferência por vaso de plantas, enquanto que na rural, dominou em recipientes dos tipos plástico e lata, na forma de lixo. Pneus albergaram a espécie nos dois ambientes.

Nos diferentes tipos de criadouros na área urbana, as larvas de Ae. aegypti foram encontradas em maior número do que as de Ae. albopictus em pneus, latas/plásticos e caixas d'água. Em vasos de plantas, as quantidades foram praticamente iguais (fig. 1). Silva \& Menezes (1996) encontraram duas larvas de Ae. scapularis em uma pequena lata junto à mata residual, na localidade de Porto Charles Nauffal, margem esquerda do rio 
Tabela III. Total de formas imaturas por espécies em diferentes tipos de criadouros, na área rural de Sertaneja, PR, entre $1^{\circ}$ de fevereiro e 24 de abril de 1995. Espécies: Aea, Ae. aegypti; Ael, Ae. albopictus; Aes, Ae. scapularis; Ana, An. argyritarsis; Ane, An. evansae; Cxb, Cx. bigoti; Cxc, Cx. sp. grupo coronator; Cxd, Cx. aff. declarator; Cxe, Cx. eduardoi; Cxh, Cx. chidesteri; Cx1, Cx. (Melanoconion) sp. 1; Cx2, Cx. (Melanoconion) sp. 2; Cxq, Cx. quinquefasciatus; Lid, Li. durhami; Psc, Ps. confinnis; Ssp, Sabethes sp.; Tsp, Toxorhynchites sp.; Url, Ur. lowii; Urn, Ur. nataliae.

\begin{tabular}{|c|c|c|c|c|c|c|c|c|c|c|c|c|c|c|c|c|c|c|c|c|}
\hline \multirow[b]{2}{*}{ Criadouros } & \multicolumn{20}{|c|}{ Espécies } \\
\hline & Aea & Ael & Aes & Ana & Ane & $C x b$ & $C x c$ & $C x d C$ & Cxe & Cxh & $C x 1$ & $C x 2$ & $C x q$ & Lid $P$ & $P_{S C} S$ & Ssp 1 & $T \mathrm{sp} L$ & Url & Urn & Total \\
\hline \multicolumn{21}{|l|}{ Artificiais } \\
\hline Pneu & 2 & 47 & & & & 4 & & & & & & & 54 & 1 & & & 1 & & & 109 \\
\hline Construção & 1 & 4 & & & & & & & & & & & & & & & & & & 5 \\
\hline Caix & & 5 & & & & & & & & & & & 29 & & & & & & & 34 \\
\hline $\begin{array}{l}\text { Peça } \\
\text { Plást }\end{array}$ & $\begin{array}{ll}o & \\
a & 7\end{array}$ & & 2 & & & 1 & & 8 & & & & & 22 & 62 & & 2 & & 3 & & 320 \\
\hline Ralo/ & & & & & & & & & & & & & & & & & & & & \\
\hline $\begin{array}{l}\text { Séptica/Vala } \\
\text { Escavação }\end{array}$ & & 25 & & & & & & & & & & & & 6 & & & & & & 31 \\
\hline $\begin{array}{l}\text { de so } \\
\text { Repre }\end{array}$ & & & & 8 & 1 & & & & & & 1 & 11 & & & 2 & & & & & 23 \\
\hline $\begin{array}{l}\text { Repre } \\
\text { Vala c }\end{array}$ & & & & & & & & & & & & & & & & & & & & \\
\hline $\begin{array}{l}\text { Drenagem } \\
\text { Natural }\end{array}$ & & & & 2 & & & & & & & & & & & & & & & & 2 \\
\hline Alagadiço & & & & 1 & 1 & & 10 & 3 & 17 & 1 & 2 & & & & & & & 13 & 31 & 79 \\
\hline Total & 10 & 300 & 2 & 11 & 6 & 4 & 10 & 12 & 17 & 1 & 3 & 11 & 105 & 69 & 2 & 2 & 1 & 13 & 31 & 610 \\
\hline
\end{tabular}

Paranapanema. Essa espécie é normalmente encontrada em coleções hídricas temporárias e semi-permanentes, formadas no solo principalmente pelas águas das chuvas (CONSOLI \& LourençO-DE-Oliveira, 1994). Aedes fluviatilis, espécie autóctone, foi encontrada em pequenos criadouros do cemitério, em cujo ambiente também foi coletada por ANDUZE (1973), SiLVA \& Lopes (1985) e Lopes et al. (1993). Estes últimos autores ressaltaram a grande capacidade de distribuição e adaptabilidade a diversos tipos de criadouros artificiais desta espécie para a área urbana de Londrina, Paraná. Este fato foi também observado no norte do Estado, antes do processo de colonização por Ae. aegypti e Ae. albopictus. Atualmente, a ausência de Ae.fluviatilis em criadouros artificiais de determinadas regiões pode estar relacionada a competitividade com outras espécies. Gonçalves, 1989, apud NATAL et al. (1997), citou ter detectado competição entre $C x$. quinquefasciatus e Ae. fluviatilis; esta última, por ser muito comum na Região Neotropical, segundo Consoli et al. (1987), é freqüentemente coletada juntamente com Cx. quinquefasciatus e Ae. aegypti.

As espécies do subgênero Culex apresentaram acentuada diversificação quanto a criadouros e ambientes. Encontradas colonizando recipientes em área urbana foram $C x$. quinquefasciatus, $C x$. aff. declarator e $C x$. sp. do grupo coronator. A primeira foi encontrada desenvolvendo-se em recipientes nos dois ambientes, sendo que, no urbano, vaso de plantas e pneu contribuíram com $63,3 \%$ das larvas coletadas e no meio rural, esteve restrita a criadouros do tipo pneu, caixa d'água, plástico e lata, quando estes estavam próximos às habitações humanas. Larvas de $C x$. aff. declarator e Culex. sp. do grupo coronator foram coletadas de recipientes em área urbana e de criadouros artificiais de solo e natural permanente, tipo alagadiço, em área rural.

As espécies encontradas apenas na área rural, colonizando coleções líquidas de 


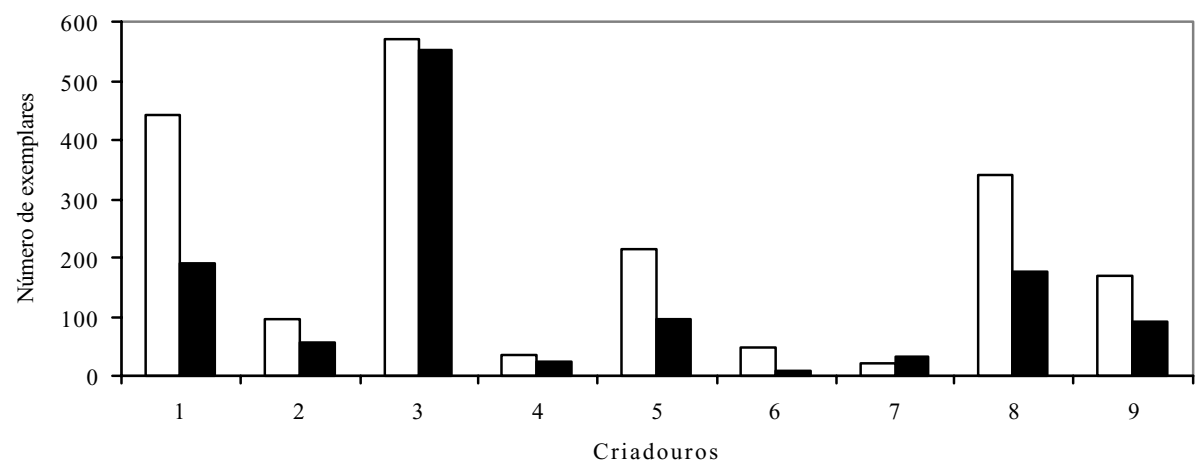

Fig. 1. Densidade de Aedes aegypti (ם) e Ae. albopictus (ם) em diferentes criadouros, na área urbana do município de Sertaneja, PR, entre 1 de fevereiro e 24 de abril de 1995. Criadouros: 1, pneu; 2, tambor/ tanque; 3, vaso de plantas; 4, material de construção; 5, caixa d'água; 6, peça de carro; 7, garrafa; 8 , plástico/lata; 9, ralo/caixa séptica/vala/poço.

solo do tipo escavação e alagadiço, foram $C x$. eduardoi, $C x$. chidesteri e Culex (Melanoconion) spp. Já Cx. bigoti foi encontrada em pneus. Limatus durhami foi mais freqüente em recipientes de plástico e lata, geralmente em locais sombreados, junto a porções de mata residual, em ambiente rural. Segundo Consoli \& L.-DE-Oliveira (1994), trata-se de um sabetíneo que aproveita recipientes abandonados em florestas, plantações geralmente próximas à mata e no peridomicílio.

Dos anofelinos coletados, An. argyritarsis foi encontrada em recipientes no ambiente urbano. No ambiente rural, esteve tanto em criadouros artificiais como naturais. A capacidade adaptativa dessa espécie a tais circunstâncias já foi mencionada por LoPEs et al. (1993). Anopheles triannulatus foi encontrada ocasionalmente no meio urbano em criadouro artificial, distintamente do citado porForATTINI (1962), que associa a sua presença a grandes coleções líquidas, expostas ao sol e com abundante vegetação horizontal e de Consolim \& FAgundes (1981) que, em coletas realizadas na região do alto rio Paraná, identificam como seus criadouros mais comuns, grandes lagoas existentes nas ilhas e nas margens do rio. Anopheles evansae foi coletada em Sertaneja no meio rural, em criadouros do tipo escavação de solo, represa e alagadiço. ForATTINI (1962) destacou como criadouros desta espécie, remansos de rios e riachos, poças, buracos em rochas, escavações diversas, como valas, barreiras, alagadiços e poços.

Encontraram-se Ur. nataliae e Ur. lowii colonizando apenas um tipo de criadouro, terreno alagadiço com água pouco profunda e coberta por vegetação. L.-DE-OLIVEIRA et al. (1986) verificaram situação similar, onde grandes massas líquidas cobertas por gramíneas corresponderam aos criadouros das espécies deste gênero.

Os vasos de plantas foram o criadouro em área urbana que apresentou maior abundância de espécies (7) e de exemplares coletados (1409), representando 35,9\% do total, seguido de pneu (19,8\%) e recipientes de plástico e lata (16,2\%). No ambiente rural, o maior percentual ficou com os recipientes de plástico e lata com 320 exemplares $(52,5 \%)$ e pneu $(17,9 \%)$. 


\section{REFERÊNCIAS BIBLIOGRÁFICAS}

Andrade, R. M. \& Rachou, R. G. 1954. Levantamento preliminar de organismos planctônicos em alguns criadouros do Anopheles darlingi no sul do Brasil. Revta bras. Malar. Doenç. trop., Rio de Janeiro, 6:481-496.

Anduze, P. J. 1973. Sobre los culicínios que procrean en receptáculos en el cemetério General del Sur de Caracas. Revta Sanid. Asist. soc. Caracas, Caracas, 5:1312-1317.

Consoli, R. A. G. B.; CAstro, M. M. T. et al. 1987. Influência da coloração do substrato no comportamento de ovoposição de Aedes fluviatilis (Lutz) (Diptera, Culicidae). Revta bras. Ent., São Paulo, 32(3-4):351-374.

Consoli, R. A. G. B. \& Lourenço-De-Oliveira, R. 1994. Principais mosquitos de importância sanitária no Brasil. Rio de Janeiro, Fiocruz. 225p.

Consolim, J. \& Fagundes, M. S. 1981. Sobre os anofelinos do rio Paraná. IV - Infecção experimental de 4 espécies com Plasmodium vivax. Arq. Biol. Tec., Curitiba, 24(4):417-423.

Forattini, O. P. 1962. Entomologia médica. São Paulo, EDUSP. v.1, 662p.

_. 1965a. Entomologia médica. São Paulo, EDUSP. v.2, 506p.

- 1965b. Entomologia médica. São Paulo, EDUSP. v.3, 416p.

. 1992. Ecologia, epidemiologia e sociedade. São Paulo, EDUSP. 529p.

Guimarães, J. H. 1997. Systematic database of Diptera of the Americas south of the United States, family Culicidae. São Paulo, Plêiade. 286 p.

Lane, J. 1953a. Neotropical Culicidae. São Paulo, Universidade de São Paulo. v. 1, 548p. 1953b. Neotropical Culicidae. São Paulo, Universidade de São Paulo. v. 2, 1112p.

Lopes, J.; Borsato, A. M. \& Pires, M. A. 1987. Entomofauna da mata Godoi. I. Culicidae (Diptera) procriando-se em criadouros artificiais introduzidos na mata. Semina, Londrina, 8(2):67-69.

Lopes, J.; Silva, M. A. N. et al. 1993. Aedes (Stegomyia) aegypti L. e a culicideofauna associada em área urbana da região Sul, Brasil. Revta Saúde Públ., São Paulo, 27(5):326-333.

Lourenço-de-Oliveira, R.; Heyden, R. \& Silva, T. F. DA. 1986. Alguns aspectos da ecologia dos mosquitos (Diptera, Culicidae) de uma área de planície (Granjas Calábria), em Jacarepaguá, Rio de Janeiro. V. Criadouros. Mems Inst. Oswaldo Cruz, Rio de Janeiro, 81(3):265-271.

Lozovei, A. L. \& Luz, E. 1976a. Diptera Culicidae em Curitiba e arredores. I. Ocorrência. Arq. Biol. Tecnol., Curitiba, 19:25-42.

1976b. Diptera Culicidae em Curitiba e arredores. II. Alimentação. Arq. Biol. Tecnol., Curitiba, 19:43-83.

Luz, E.; Consolim, J. et al. 1987. Larvas de Anopheles (subgênero Kerteszia) Theobald, 1905 encontradas em criadouros artificiais no Estado do Paraná, Brasil. Revta Saúde Públ., São Paulo, 21(5):466-468.

Natal, D.; Gonçalves, E. F. D. \& Taveira, L. A. 1997. Proliferação de mosquitos (Diptera; Culicidae) em cemitérios e perspectivas de controle. Informe Epid. do SUS, Brasília, 6(2):103-110.

Reinert, J. F. 1975. Mosquitoes generic and sub generic abreviations (Diptera: Culicidae). Mosq. Systematics, Baltimore, 7(2):105-110.

Silva, M. A. N. \& Lopes, J. 1985. Dados sobre a potencialidade criadoura de Culicidae (Diptera) do Cemitério São Pedro - Londrina - Paraná. Semina, Londrina, 6(3):133-139.

Silva, A. M. \& Menezes, R. M. T. 1996. Encontro de Aedes scapularis (Diptera: Culicidae) em criadouro artificial em localidade da região Sul do Brasil. Revta Saúde Públ., São Paulo, 30(1):103-104.

Wons, I. 1985. Geografia do Paraná. 5ª ed. Curitiba, Ensino Renovado. 172p.

Recebido em 25.09.2000; aceito em 22.08.2002.

Iheringia, Sér. Zool., Porto Alegre, 92(4):31-36, 30 de dezembro 2002 